\title{
Sentinel lymph node biopsy reduces the incidence of secondary neck metastasis in patients with oral squamous cell carcinoma
}

\author{
AKIMITSU HIRAKI ${ }^{1}$, DAIKI FUKUMA ${ }^{2}$, MASASHI NAGATA $^{2}$, SHINYA SHIRAISHI $^{3}$, KENTA KAWAHARA $^{2}$, \\ YUICHIRO MATSUOKA ${ }^{2}$, YOSHIHIRO NAKAGAWA ${ }^{2}$, RYOJI YOSHIDA ${ }^{2}$, TAKUYA TANAKA ${ }^{2}$, \\ YOSHIHIRO YOSHITAKE ${ }^{4}$, MASANORI SHINOHARA $^{4}$, YASUYUKI YAMASHITA ${ }^{3}$ and HIDEKI NAKAYAMA ${ }^{2}$ \\ ${ }^{1}$ Division of Oral Oncology, Department of Oral and Maxillofacial Surgery, Fukuoka Dental College, \\ Fukuoka 814-0193; Departments of ${ }^{2}$ Oral and Maxillofacial Surgery, Sensory and Motor Organ Sciences \\ and ${ }^{3}$ Diagnostic Radiology, Faculty of Life Sciences, Kumamoto University, Kumamoto \\ 860-8555; ${ }^{4}$ Itoh Dento-Maxillofacial Hospital, Kumamoto 860-8556, Japan
}

Received December 23, 2015; Accepted April 15, 2016

DOI: $10.3892 / \operatorname{mco} .2016 .882$

\begin{abstract}
It has recently been established that sentinel node biopsy (SNB) is an applicable and feasible procedure for the prediction of neck lymph node status in patients with early oral squamous cell carcinoma (OSCC) who are clinically negative for neck metastasis (cN0). The aim of this study was to retrospectively compare excision followed by watchful waiting with excision and SNB, in order to determine the effectiveness of SNB. A total of 125 patients with cN0 early OSCC were divided into two groups, namely the excision alone $(n=78)$ and excision with SNB ( $n=47)$ groups. The clinical data of these two groups between 2006 and 2013 were analyzed. In the excision with SNB group, the negative predictive value and false-negative rate of SNB were 94\% (30/32) and 18\% (2/11), respectively. Secondary neck metastasis, also known as delayed neck metastasis, occurred in $24.2 \%$ of the patients in the excision alone group and $4.9 \%$ of the patients in the excision with SNB group. The 5-year overall survival (OS) rates were 84.0 and $97.5 \%$ in the excision alone and excision with SNB groups, respectively. Significant differences were found in the rate of secondary neck metastasis and OS between the
\end{abstract}

Correspondence to: Dr Akimitsu Hiraki, Division of Oral Oncology, Department of Oral and Maxillofacial Surgery, Fukuoka Dental College, 2-15-1 Tamura, Sawara-ku, Fukuoka 814-0193, Japan

E-mail: hiraki@college.fdcnet.ac.jp

Abbreviations: CT, computed tomography; END, elective neck dissection; FDG-PET, fluorodeoxyglucose positron emission tomography; MRI, magnetic resonance imaging; MRND, modified radical neck dissection; NPV, negative predictive value; OS, overall survival; OSCC, oral squamous cell carcinoma; RND, radical neck dissection; SN, sentinel node; SNB, sentinel node biopsy; US, ultrasound

Key words: secondary neck metastasis, delayed neck metastasis, oral squamous cell carcinoma, sentinel lymph node, biopsy, watchful waiting, elective neck dissection two groups. SNB may be effective in the detection of occult neck lymph node metastasis, with a reduction in the incidence of secondary neck metastasis and improvements in the 5-year OS in patients with early-stage (stage I/II) oral cancer.

\section{Introduction}

The status of lymph nodes in the neck is the most important prognostic factor for oral squamous cell carcinoma (OSCC). The rate of secondary neck metastases of OSSC is 20-30\% (1-6). Even with advanced modern imaging techniques, including computed tomography (CT), magnetic resonance imaging (MRI), ultrasound (US) and fluorodeoxyglucose positron emission tomography (FDG-PET), the accuracy of current diagnostic imaging does not exceed $80 \%$ (2).

The treatment strategies for early-stage oral cancer clinically negative for neck metastasis (cN0) include performing elective neck dissection (END) or opting for the watchful waiting approach. END is currently the standard treatment for patients in the absence of positive lymph nodes on imaging tests. Some reports recommend END, as it reduces the risk of uncontrolled disease $(2,3)$. Furthermore, a recent meta-analysis of randomized controlled trials suggested a survival advantage with END (7). However, $\leq 70 \%$ of patients undergo unnecessary neck dissection, resulting in decreased quality of life $(8,9)$.

Therefore, accurate detection of occult metastases is crucial, as it allows for appropriate treatment planning.

With respect to sentinel node biopsy (SNB) in oral cancer, large multi-institutional clinical trials have been conducted $(10,11)$ and numerous studies have demonstrated that SNB is associated with predictably high accuracy in identifying occult metastasis $(10,12)$.

The objective of this study was to determine the benefits of SNB for cNO disease. We retrospectively compared long-term survival following excision alone or excision and SNB in patients with OSCC. Moreover, we analyzed the complications and surgical stress of the SNB procedure, including limited range of motion in the arm, size of the surgical scar and operative time. 


\section{Patients and methods}

Patient characteristics. A total of 125 patients with OSCC clinically staged as N0 underwent tumor excision with/without SNB at the Department of Oral and Maxillofacial Surgery of Kumamoto University (Kumamoto, Japan) from 2006 to 2013. SNB was performed in patients who consented to the procedure. The excision alone group included 78 patients, and the excision with SNB group included 47 patients. All the patients were diagnosed with a primary oral tumor, and no patients had received prior radiotherapy or chemotherapy.

Diagnostic imaging specialists comprehensively diagnosed lymph node status with imaging techniques, including CT, MRI, US and FDG-PET.

The study included 84 men and 41 women, with a mean age of 65.6 years (range, 28-85 years). The primary tumor sites included the tongue $(n=79)$, lower gingiva $(n=22)$, upper gingiva $(n=10)$, buccal mucosa $(n=8)$ and oral floor $(n=6)$. Regarding T-classification, 48 cases were $\mathrm{T} 1$ and 77 were T2. The median postoperative follow-up period was $43.6 \pm 24.7$ months. Postoperative radiotherapy was administered to 4 patients in the excision alone group and 2 patients the excision with SNB group. The patient characteristics did not differ significantly between the excision alone and the excision with SNB groups (Table I).

Surgical procedure. On the day before surgery, the patients received injections of $37 \mathrm{MBq} 99 \mathrm{mTc}$-phytate into 4 selected sites of the submucosal layer surrounding the tumor.

Sentinel nodes (SNs) were identified by single-photon emission $\mathrm{CT} / \mathrm{CT}$ after 2 hours, and the operator confirmed the anatomical position and number of nodes. At the time of surgery, the SNs were removed with a hand-held gamma detector probe. During the operation, all SNs were cut in $4-\mu \mathrm{m}$ sections at 2-mm intervals and examined by hematoxylin and eosin staining. In cases for which the rapid intraoperative diagnosis was positive, radical neck dissection (RND) or modified radical neck dissection (MRND) was performed. These SNs were further examined by pathological investigation of paraffin-embedded sections.

Following discharge from the hospital, the patients in the two groups were followed up every 2 weeks and carefully examined by CT, MRI and US every 3 months.

We determined the number and distribution of the detected SNs according to the American Academy of Otolaryngology-Head and Neck Surgery classification (13). The secondary neck metastasis rate, negative predictive value (NPV) and false-negative rate of SNB were calculated (TN, $30 \mathrm{pts}) /(\mathrm{FN}+\mathrm{TN}, 32 \mathrm{pts})$ and the false-negative rate as (FN, 2 pts)/(TP + FN, 11 pts). Furthermore, overall survival (OS) was compared between the excision alone and excision with SNB groups.

In the excision with SNB group, we measured the time required for the removal of SNs and the length of the surgical scar, and assessed the surgical stress associated with the SNB procedure.

Statistical analysis. All statistical analyses were performed using the JMP9 statistical software (SAS Institute Inc., Cary, NC, USA). The secondary metastasis rates of the two groups were compared using the Chi-squared test. The 5-year OS rates were estimated using the Kaplan-Meier method and the log-rank test was used for univariate analysis. P-values $<0.05$ were considered to indicate statistically significant differences.

Our retrospective study and clinical record reviews were approved by the Review Board of our institution. Written informed consent was obtained from all study patients. This investigation was conducted according to the principles of the Helsinki Declaration.

\section{Results}

SN detection. The SN detection rate was $100 \%$ and the average number of removed SNs was 2.1 per patient (range, 1-5 nodes). In $46 / 47$ patients, only ipsilateral SNs were removed. On the ipsilateral side, 46 SNs were located at level I, 38 at level II, 10 at level III, 2 at level IV, and 1 at level V. On the contralateral side, $1 \mathrm{SN}$ was located at level II and $1 \mathrm{SN}$ was located at level III.

Follow-up. In the excision alone group $(\mathrm{n}=78), 12$ local recurrences of primary tumor and 16 secondary neck metastases occurred during the follow-up period. Conversely, in the excision with SNB group $(n=47)$, intraoperative histopathological examination of the SNs revealed micrometastases in 9 patients; 6 local recurrences of primary tumor occurred, and secondary neck metastasis appeared in 2 cases during the follow-up period. An RND or MRND was performed in $9 \mathrm{SN}$-positive cases, and no further metastatic lymph nodes were isolated from the dissected neck tissue. The NPV, the false-negative rates and the SNB were calculated while excluding local recurrences of primary tumor. The NPV and false-negative rate of SNB were 94\% (30/32) and 18\% (2/11), respectively (Fig. 1). The rate of secondary neck metastasis was calculated. The rate of secondary neck metastasis in the excision with SNB group was lower compared with that in the excision alone group ( 4.9 vs. $24.2 \%$, respectively; $\mathrm{P}<0.05$ ).

The number of deaths in the excision alone and excision with SNB groups was 12 and 1, respectively. The 5-year OS rates for the excision alone and excision with SNB groups were 84.0 and $97.5 \%$, respectively $(\mathrm{P}<0.05)$ (Fig. 2).

In the excision with SNB group, the time required to remove the SNs was 9.2 min per $\mathrm{SN}$, and the length of the surgical scar was $53 \mathrm{~mm}$. The surgical stress associated with SNB was minimal, and no complications, such as neck hematoma, facial palsy, postoperative infection, or limited range of motion in the arm, were observed.

\section{Discussion}

It has been well established that the presence or absence of lymph node metastasis in the neck is the most important prognostic factor for OSCC.

The prevalence of neck metastasis in cN0 early oral carcinoma is relatively high, with rates ranging from 20 to $30 \%(2,12)$. However, if all cNO oral carcinoma patients underwent END, this would result in overtreatment of patients without metastasis. Currently, it remains controversial whether END is necessary for the optimal management of patients clinically negative for neck metastasis. Several reports have recommended END, but there has been no definitive conclusion (14-17). 
Table I. Patient characteristics.

\begin{tabular}{|c|c|c|c|}
\hline Characteristics & $\begin{array}{l}\text { Excision alone } \\
\text { group }(n=78)\end{array}$ & $\begin{array}{l}\text { Excision with SNB } \\
\text { group }(n=47)\end{array}$ & P-value \\
\hline Gender & & & $0.31^{\mathrm{a}}$ \\
\hline Male, $\mathrm{n}$ & 55 & 29 & \\
\hline Female, $\mathrm{n}$ & 23 & 18 & \\
\hline Age, years & & & $0.97^{\mathrm{b}}$ \\
\hline Mean \pm SD & $65.4 \pm 12.6$ & $65.5 \pm 13.0$ & \\
\hline Primary site & & & $0.94^{\mathrm{a}}$ \\
\hline Tongue & 50 & 29 & \\
\hline Lower gingiva & 13 & 9 & \\
\hline Upper gingiva & 7 & 3 & \\
\hline Buccal mucosa & 5 & 3 & \\
\hline Oral floor & 3 & 3 & \\
\hline T-classification & & & $0.12^{\mathrm{a}}$ \\
\hline 1 & 34 & 14 & \\
\hline 2 & 44 & 33 & \\
\hline Follow-up period, months & & & $0.07^{\mathrm{b}}$ \\
\hline Mean \pm SD & $46.6 \pm 23.2$ & $38.5 \pm 26.1$ & \\
\hline Postoperative radiotherapy & & & $0.82^{\mathrm{a}}$ \\
\hline Yes, $\mathrm{n}$ & 4 & 2 & \\
\hline No, n & 74 & 45 & \\
\hline
\end{tabular}

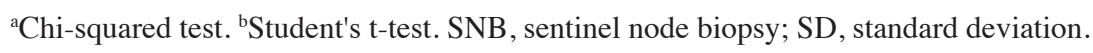

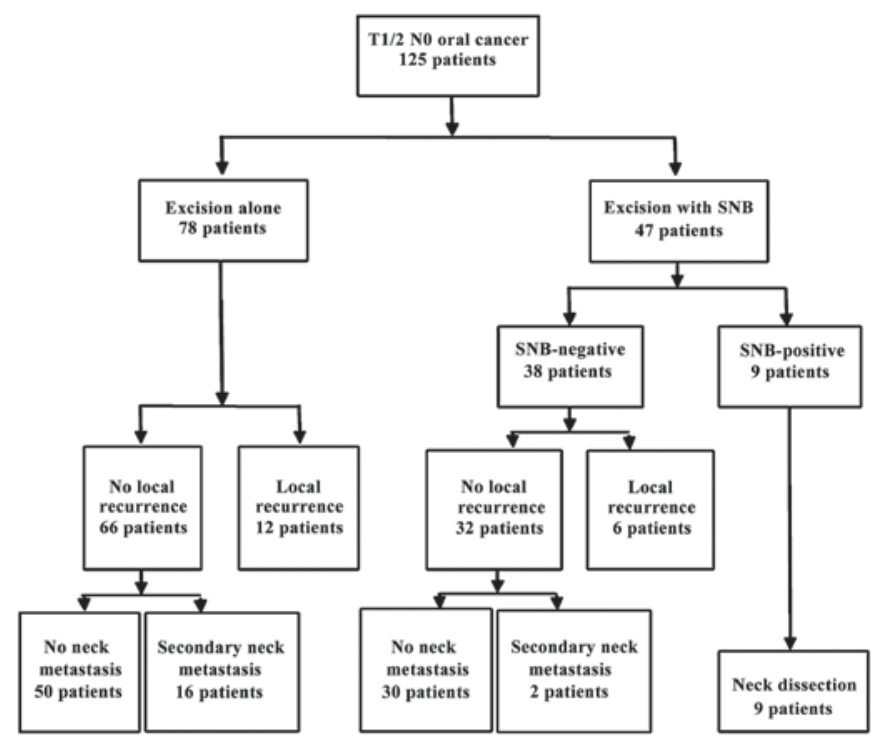

Figure 1. Overview of treatment course. Study flow and follow-up of patients with T1/2 N0 oral cancer in the excision alone and excision with SNB groups. SNB, sentinel node biopsy.

SNB has attracted attention as a new diagnostic tool that allows for the detection of micrometastasis in the neck lymph nodes.

In multi-institutional trials, SNB has been shown to exhibit a predictably high accuracy for identifying occult metastasis $(3,10,14,18,19)$.

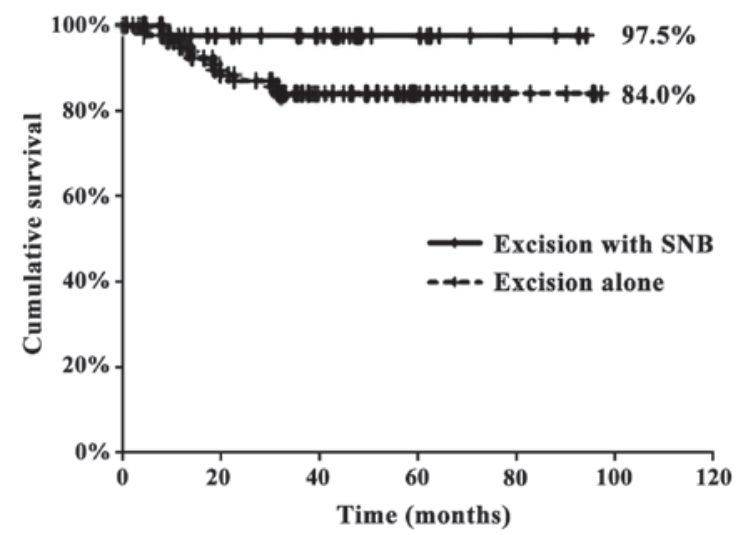

Figure 2. Five-year overall survival. There was a significant difference in overall survival between the excision alone (dotted line) and excision with SNB (solid line) groups $(\mathrm{P}<0.05)$.

A previous study by the American College of Surgeons Oncology Group reported an NPV of $94 \%$ and a false-negative rate of $7 \%$ (8). In other reports, the NPV and false-negative rates were $90-96$ and $2.5-22 \%$, respectively $(3,10,18,20,21)$. In the present study, the NPV and false-negative rates were 94 and $18 \%$, respectively, which were similar to previously reported rates. Thus, performing SNB is feasible, and this procedure may be used to accurately predict neck metastasis in $\mathrm{cNO}$ OSCC patients.

In our department, we have adopted the watchful waiting approach to manage $\mathrm{cNO}$ early OSCC. If the occurrence of 
secondary neck metastasis becomes clinically apparent, RND or MRND is immediately performed. Therefore, it is very important to detect secondary metastases early, as this is directly associated with patient prognosis. The patients were followed up every 2 weeks, and carefully evaluated using CT, MRI and US every 3 months.

In the present study, SNs were detected in all the cases, and the distribution of SNs was consistent with previous reports (2). While evaluating neck dissection tissue in the $9 \mathrm{SN}$-positive cases, we identified no metastasis other than the one in the SN. On the basis of these results, evaluating the SNs in oral cancer appears to be applicable and feasible for the prediction of the status of neck lymph nodes in cNO early OSCC.

The rates of secondary neck metastasis in the excision alone and excision with SNB groups were 4.9 and 24.2\%, respectively, and this difference was statistically significant. Other reports have demonstrated that secondary neck metastasis occurred in $0-6 \%$ of cases that underwent SNB, and our results were similar $(3,18,20,21)$.

Our study observed 5-year OS rates of 84.0 and $97.5 \%$ for the excision alone and excision with SNB groups, respectively, and this difference was statistically significant. Thus, SNB reduced the rate of secondary neck metastasis and increased the 5-year OS rate. Other studies reported a 5-year OS rate of $80 \%$ and a 2 -year OS rate of $87-90 \%$; our results are superior to those findings $(3,18,20,21)$, suggesting that SNB is an effective approach in cN0 early OSCC. However, there have been few previous reports comparing excision alone to excision with SNB. Yamauchi et al reported that the rate of secondary metastasis in the watchful waiting and SNB groups was 27 and $9.1 \%$, respectively, and this difference was not statistically significant (21).

Our study included only a limited number of cases; therefore, further large multi-institutional clinical trials are required to investigate the long-term prognosis of patients who undergo SNB.

In this study, the surgical stress and postoperative complications of the SNB procedure were minimal. In this respect, our findings were similar to those of other studies reporting that postoperative complications of neck dissection, such as neck hematoma, facial palsy, postoperative infection and limited range of motion in the arm, rarely occur after SNB (20).

In conclusion, SNB is a minimally invasive and highly reliable staging method, reduces the rate of secondary neck metastasis and improves 5-year OS in patients with stage I and II oral cancer.

\section{Acknowledgements}

We would like to thank Editage (www.editage.jp) for the English language editing.

\section{References}

1. Bilde A, von Buchwald C, Therkildsen MH, Mortensen J, Kirkegaard J, Charabi B and Specht L: Need for intensive histopathologic analysis to determine lymph node metastases when using sentinel node biopsy in oral cancer. Laryngoscope 118: 408-414, 2008.

2. Hernando J, Villarreal P, Álvarez-Marcos F, Gallego L, García-Consuegra L and Junquera L: Comparison of related complications: Sentinel node biopsy versus elective neck dissection. Int J Oral Maxillofac Surg 43: 1307-1312, 2014.
3. Samant S: Sentinel node biopsy as an alternative to elective neck dissection for staging of early oral carcinoma. Head Neck 36: 241-246, 2014.

4. Sloan P: Head and neck sentinel lymph node biopsy: Current state of the art. Head Neck Pathol 3: 231-237, 2009.

5. Stoeckli SJ, Alkureishi LW and Ross GL: Sentinel node biopsy for early oral and oropharyngeal squamous cell carcinoma. Eur Arch Otorhinolaryngol 266: 787-793, 2009.

6. von Buchwald C, Bilde A, Shoaib T and Ross G: Sentinel node biopsy: The technique and the feasibility in head and neck cancer. ORL J Otorhinolaryngol Relat Spec 64: 268-274, 2002.

7. Fasunla AJ, Greene BH, Timmesfeld N, Wiegand S, Werner JA and Sesterhenn AM: A meta-analysis of the randomized controlled trials on elective neck dissection versus therapeutic neck dissection in oral cavity cancers with clinically node-negative neck. Oral Oncol 47: 320-324, 2011.

8. Kuntz AL and Weymuller EA Jr: Impact of neck dissection on quality of life. Laryngoscope 109:1334-1338, 1999.

9. Nibu KI, Ebihara Y, Ebihara M, Kawabata K, Onitsuka T, Fujii T and Saikawa M: Quality of life after neck dissection: A multicenter longitudinal study by the Japanese Clinical Study Group on Standardization of Treatment for Lymph Node Metastasis of Head and Neck Cancer. Int J Clin Oncol 15: 33-38, 2010.

10. Civantos FJ, Zitsch RP, Schuller DE, Agrawal A, Smith RB, Nason R, Petruzelli G, Gourin CG, Wong RJ, Ferris RL, et al: Sentinel lymph node biopsy accurately stages the regional lymph nodes for T1-T2 oral squamous cell carcinomas: Results of a prospective multi-institutional trial. J Clin Oncol 28: 1395-1400, 2010.

11. Yuen AP, Wei WI, Wong YM and Tang KC: Elective neck dissection versus observation in the treatment of early oral tongue carcinoma. Head Neck 19: 583-588, 1997.

12. Stoeckli SJ: Sentinel node biopsy for oral and oropharyngeal squamous cell carcinoma of the head and neck. Laryngoscope 117: 1539-1551, 2007.

13. Robbins KT, Clayman G, Levine PA, Medina J, Sessions R, Shaha A, Som P and Wolf GT; American Head and Neck Society; American Academy of Otolaryngology-Head and Neck Surgery: Neck dissection classification update: Revisions proposed by the American Head and Neck Society and the American Academy of Otolaryngology-Head and Neck Surgery. Arch Otolaryngol Head Neck Surg 128: 751-758, 2002

14. Alvarez J, Bidaguren A, McGurk M, Diaz-Basterra G, Brunsó J, Andikoetxea B, Martín JC, Barbier L, Arteagoitia I and Santamaría JA: Sentinel node biopsy in relation to survival in floor of the mouth carcinoma. Int J Oral Maxillofac Surg 43: 269-273, 2014.

15. Bessell A, Glenny AM, Furness S, Clarkson JE, Oliver R, Conway DI, Macluskey M, Pavitt S, Sloan P and Worthington HV: Interventions for the treatment of oral and oropharyngeal cancers: Surgical treatment. Cochrane Database Syst Rev: Cd006205, 2011.

16. Keski-Säntti H, Atula T, Törnwall J, Koivunen P and Mäkitie A: Elective neck treatment versus observation in patients with T1/T2 N0 squamous cell carcinoma of oral tongue. Oral Oncol 42: 96-101, 2006

17. Rodrigo JP, Shah JP, Silver CE, Medina JE, Takes RP, Robbins KT, Rinaldo A, Werner JA and Ferlito A: Management of the clinically negative neck in early-stage head and neck cancers after transoral resection. Head Neck 33: 1210-1219, 2011.

18. Alkureishi LW, Ross GL, Shoaib T, Soutar DS, Robertson AG, Thompson R, Hunter KD, Sorensen JA, Thomsen J, Krogdahl A, et al: Sentinel node biopsy in head and neck squamous cell cancer: 5-year follow-up of a European multicenter trial. Ann Surg Oncol 17: 2459-2464, 2010.

19. Ross GL, Soutar DS, Gordon MacDonald D, Shoaib T, Camilleri I, Roberton AG, Sorensen JA, Thomsen J, Grupe P, Alvarez J, et al: Sentinel node biopsy in head and neck cancer: Preliminary results of a multicenter trial. Ann Surg Oncol 11: 690-696, 2004.

20. Schiefke F, Akdemir M, Weber A, Akdemir D, Singer S and Frerich B: Function, postoperative morbidity, and quality of life after cervical sentinel node biopsy and after selective neck dissection. Head Neck 31: 503-512, 2009.

21. Yamauchi K, Fujioka Y and Kohno N: Sentinel node navigation surgery versus observation as a management strategy for early tongue carcinoma. Head Neck 34: 568-572, 2012. 